\title{
On quality of NKG2015 geoid model over the Nordic countries
}

DOI: https://doi.org/10.1515/jogs-2019-0010

Received July 15, 2018; accepted October 29, 2019

\begin{abstract}
The NKG2015 geoid model covers the Nordic and Baltic countries and has been computed based on the least-squares modification of Stokes' formula with additive corrections method. New and precise terrestrial, airborne and shipborne gravimetric measurements, the recent global gravity model of the gravity field and steadystate ocean circulation explorer (GOCE) and detailed digital terrain models over each territory have been used for computing this new geoid model. Some estimates for the error of this model have been roughly presented by comparing it with the global navigation satellite system (GNSS) data over each country. In this paper, our goal is to have a closer look at the relative error of this model by performing some statistical tests and finding the proper corrective surface for absorbing the systematic errors over each country. Our main assumption is realisticity of the errors of GNSS/levelling data and we will investigate its consequences in estimating the error of the geoid model. Our results show that the 4-parameter corrective surface is suitable for modelling the systematic trends of the differences between the gravimetric and GNSS geoid heights in Sweden, Denmark and Finland, but a filtered discrepancies by a confidence interval of $95 \%$ should be used for Sweden. A 7-aparameter model is suitable for the filtered discrepancies with the confidence interval of $95 \%$ in Norway. Based on the selected corrective surface and our newly developed regional iterative variance estimator, the confidence interval for the error of NKG2015 geoid model in Sweden, Denmark and Norway yielded 0-6.5 mm, 1.8-5.2 mm, 14.8-17.7 $\mathrm{mm}$, respectively with a confidence level of $95 \%$. We could not estimate the geoid error in Finland because the given error of the GNSS/levelling heights is significantly larger than the size of residuals. Based on the selected corrective surfaces and our presented local variance estimator, the average error of geoid becomes 3.6, 2.4, 8.8 and $5.8 \mathrm{~mm}$ with a confidence interval of $68 \%$, respectively, over Sweden, Denmark, Norway and Finland.
\end{abstract}

*Corresponding Author: Mehdi Eshagh: Department of Engineering Science, University West, Sweden, E-mail: mehdi.eshagh@hv.se Jenny Berntsson: Department of Engineering Science, University West, Sweden
Keywords: estimation, GNSS/levelling, hypothesis testing, normality, confidence interval, variance

\section{Introduction}

The geoid is an equipotential surface, which has the best fit to mean sea level and continues beneath the continents. It is a reference for heights and called vertical datum in geodetic literature as well. Lateral variation of this surface represents changes in the Earth's gravity field due to mountains, valleys, oceans as well as the Earth's interior structure and density. Therefore, the Earth's gravity field and information about the density and height of topographic masses can be used for computing a geoid model. Today, according to advances in technology, the gravimetric measurements are collected with high qualities and spatial resolution. This makes the role of geoid even more important than before, and it is necessary to compute new geoid models whenever new data become available. Definitely, the qualities of the new models increase, and it is important to check such qualities prior to using the models.

The geoid can be used for solving the levelling problems. We know that levelling is a very time consuming and costly work. However, measuring the geodetic/ellipsoidal height by a global navigation satellite system (GNSS) receiver is much easier in practice, but the measured height is referred to the reference ellipsoid and not the geoid. We already know the relation between the geodetic, orthometric and geoid heights:

$$
N=h-H
$$

where $N$ is the geoid height, $H$ the orthometric height and $h$ the ellipsoidal/geodetic height, which is measured by a GNSS receiver. If two of these heights are known, the other one can be computed. For example, if $N$ has already been determined and $h$ is measured by GNSS receivers, $H$ can be determined, then, there will not be any need for performing the time consuming and costly sprit levelling. This method of height determination is called GNSS/levelling (GL).

The newest geoid model developed by the Nordic Geodetic Commission (NKG) was named NKG2015 geoid 
model (Ågren et al. 2016). It is of importance to see how close this model is to the geoid heights computed from GL data. The NKG2015 geoid model is the most recent official geoid model, which has been developed specifically for the Nordic countries Sweden, Norway, Finland and Denmark and some Baltic countries. It has been computed based on inland terrestrial gravimetric data, airborne/shipborne gravity data for the seas. The satellite gravity model GO_CONS_GCF_2_DIR_R5 (Bruinsma et al. 2013) to degree and order 300 , has been used to present the long wavelength portion of the geoid model. This model is a satellite-only model based on a full combination of the gravity field and steady-state ocean circulation explorer (GOCE) (ESA 1999). The combined national topographic heights with those of Satellite Radar Topography Mission (SRTM3) (Farr et al. 2007) with the resolution 3 second and GTOPO30 as well as some ice models over Norway have been considered in geoid modelling. Amongst different methods of geoid determination, which were tested and applied, the unbiased type of least-squares modification of Stokes' formula with additive corrections was selected as the best method. This method has been developed at the Royal Institute of Technology (KTH) during years by Lars E. Sjöberg, and practically developed by Ågren (2004) and Ellmann $(2004,2005)$ and applied by Kiamehr (2006), Daras (2009), Abdalla (2009), Ulotou (2009) and Ssegendo (2016) successfully over different regions and countries. Some aspect about gravity survey in the geoid modelling process can be found in Märdla et al. (2017). The National Land Survey of Sweden has adopted this method for geoid determination.

Quality of a geoid model is usually tested by comparing it with the GL data. Fotopoulos (2003 and 2005) used variance component estimation to calibrate the error of geoid models and GL data. She considered different corrective surfaces to model the discrepancies between geoid models and the geoid computed from the GL data. Full variance-covariance matrices of geoidal, orthometric and ellipsoidal heights were considered in her analyses. Such matrices can be obtained in different ways; for more details see Fotopoulos (2003 and 2005). Other similar works were carried out by Kiamehr and Eshagh (2008) and Eshagh and Sjöberg (2008) for calibrating the error of the gravimetric geoid of Iran. They did not use the full variance-covariance matrices of the heights and used the as initial values of variance components, but they empirically estimated the error of the geoid, orthometric and ellipsoidal heights and used variance component estimation. Eshagh (2010) calibrated the error of the KTH08 geoid model (Ågren et al., 2009) using this method over Sweden. Another way for estimation of variance is to use the a pos- teriori variance factor directly, instead of variance component, if the error of some measurements are trustful. Wagner and McAdoo (2012) used this process to calibrate the gravity models and Eshagh (2013) used a similar method to calibrate the errors of the recent gravity field models based on their differences with respect to EGM08 (Pavlis et al. 2012).

There are, however, previous quality analysis research on other geoid models, e.g. Eshagh (2010) performed a similar study in Sweden based on the KTH08 geoid model (Ågren et al. 2009). Zoghi (2015) and Eshagh and Zoghi (2016) analysed the quality of the geoid model, computed from the global gravity model EGM08 over the Nordic countries. In addition, the working group on geoid and height system determination of the NKG has specified new sets of GL heights for evaluation of the geoid models over the Nordic countries. Varbla et al. (2017) evaluated different marine geoid models including the NKG2015 model, by ship-borne Global Navigation Satellite Systems (GNSS) profiles. Ellmann et al. (2019) has also determined a geoid model over Estonia and evaluated it by an innovative twostage stochastic fit based on least-squares collocation using these GL heights and estimated an accuracy of $5 \mathrm{~mm}$ for their geoid model. Here, our goal is to use these GL heights for evaluation of the NKG2015 geoid model in a different way by using corrector surfaces and variance estimation.

\section{Error of geoid based on GNSS/levelling heights and discrepancies}

Equation (1) shows the ideal relation between the ellipsoidal, orthometric and geoid heights. This equation is theoretically valid, but due to the presence of different errors and biases in the heights this is not so. To be more realistic we should write Eq. (1) in the following form:

$$
h+\varepsilon_{h}-\left(H+\varepsilon_{H}\right)-\left(N+\varepsilon_{N}\right)=w,
$$

where $\varepsilon_{h}$ and $\varepsilon_{H}$ are the random error of the ellipsoidal and normal heights with the property $\mathrm{E}\left\{\varepsilon_{h}\right\}=\mathrm{E}\left\{\varepsilon_{H}\right\}=0$, with $\mathrm{E}\{\}$ denoting the statistical expectation operator. In addition, $\mathrm{E}\left\{\varepsilon_{h} \varepsilon_{h}\right\}=\sigma_{h}^{2}$ and $\mathrm{E}\left\{\varepsilon_{H} \varepsilon_{H}\right\}=\sigma_{H}^{2}$ as variances of the heights. The symbol $w$ stands for discrepancies of the equation with $\mathrm{E}\{w\}=0$.

Eq. (2) is a condition adjustment model, in such a model the a posteriori variance factor can be estimated by:

$$
\hat{\sigma}_{0}^{2}=\frac{w^{2}}{\sigma_{h-H}^{2}+\sigma_{N}^{2}} .
$$


After solving Eq. (3) for $\sigma_{N}^{2}$ we obtain:

$$
\sigma_{N}=\sqrt{\frac{w^{2}}{\hat{\sigma}_{0}^{2}}-\sigma_{h-H}^{2}} .
$$

Equation (4) has a real solution if:

$$
\frac{w^{2}}{\hat{\sigma}_{0}^{2}} \geq \sigma_{h-H}^{2} .
$$

This means that the ratio of squared of a discrepancy and a posteriori variance factor should be larger than the variance of the subtraction of the normal (orthometric) and ellipsoidal heights. However, the value of the a posterior variance factor is not given so that Eq. (5) can be applied for finding the error of the geoid height. In addition, we know that in an ideal case it should be equal to 1 , when the mathematical model is suitable for modelling the data, no gross error exists on the data and the considered error for the data is in agreement with discrepancies.

As Eq. (5) shows $w$ plays a significant role in estimation of the error of the geoid heights. Therefore, performing some statistical tests on it is of vital importance for a realistic estimation of the geoid height error.

\section{Test of discrepancies}

Discrepancies should be checked to see if they are normally distributed or contain any systematic trend. In the following, we will present filtering of the discrepancies based on confidence intervals (CIs) of $95 \%$ and $99 \%$, chisquare goodness-of-fit (GOF) test, and test of skewness and kurtosis of distribution of $w$.

\subsection{Filtering of discrepancies based on $95 \%$ and $99 \%$ confidence intervals}

Here, values of $w$ are computed for all points and their mean $\bar{w}$ and standard deviation $s_{w}$ are used for filtering the data with CIs of $95 \%$ and $99 \%$. Generally, this CI can be written in the following form:

$$
\bar{w}-Z_{\alpha / 2} s_{w} \leq w \leq \bar{w}+Z_{\alpha / 2} s_{w}
$$

where for a CI of $95 \%$, the significant level will be $5 \%$ and according to the normal standard distribution we have $Z_{0.025}=1.96$ and for $99 \%$ and $1 \%$, we have $Z_{0.005}=3$. This means that each value of $w$ should be inside the interval (6), otherwise, it is recognised as an outlier, and should be removed from the set of $w$.

\subsection{Chi-square goodness-of-fit test}

Histogram of $w$ can give us some idea about the normality visually. Histogram of normally distributed data should be bell-shaped and symmetric. However, if the goal is to check the normality of $w$ by their histogram in a more complete way, the chi-square GOF should be applied. The statistic, which is used for such a test, is:

$$
y=\sum_{i=1}^{n b} \frac{\left(O_{i}-E_{i}\right)^{2}}{E_{i}} \leq \chi_{\alpha, n b-1}^{2}
$$

where $i$ stands for counter of histogram classes or bins and $n b$ is the total number of bins, and $O_{i}$ the measured frequency of the measurement in each bin and $E_{i}$ the expected frequency, which is estimated from a normal distribution function, computed based the sample mean and standard deviation. $\chi_{\alpha, n b-1}^{2}$ is the number that we find from the chi-square distribution function with significant level of $\alpha$ and $n b-1$ degree of freedom. The computed statistic $y$ should be smaller than this value, if this is the case, $w$ has probably normal distribution.

\subsection{Test of skewness and kurtosis of histogram}

Generally, skewness is a factor showing lack of symmetry. A symmetric dataset looks the same to the left and right of the centre point. However, it can be skewed to the left and right of this point depending on the sign of skewness. Kurtosis is a factor showing if the data are heavy-tailed or lighttailed. That is, data sets with high kurtosis tend to have heavy tails, or outliers. Data sets with low kurtosis tend to have light tails, or lack of outliers. Normal distribution has zero skewness and kurtosis.

The skewness $S$ and kurtosis $K$ of univariate data $w_{i}$, $i=1,2, . ., n$, where $n$ is total number of the GL points are, respectively,

$$
\begin{gathered}
S=\frac{n}{s_{w}^{3}} \sum_{i=1}^{n}\left(w_{i}-\bar{w}\right)^{3} \\
K=\frac{n}{s_{w}^{4}} \sum_{i=1}^{n}\left(w_{i}-\bar{w}\right)^{4}-3 .
\end{gathered}
$$

The variance of skewness and kurtosis are, respectively, approximated by $6 / n$ and $24 / n$. The CIs for the standardised skewness and kurtosis are:

$$
\begin{aligned}
& -Z_{\alpha / 2} \leq \frac{S}{\sigma_{S}} \leq Z_{\alpha / 2} \\
& -Z_{\alpha / 2} \leq \frac{K}{\sigma_{K}} \leq Z_{\alpha / 2} .
\end{aligned}
$$


If the standardised skewness and kurtosis are inside the CI, then $w$ has probably normal distribution.

\section{Least-squares detrending of discrepancies and statistical tests}

The discrepancies $w$ may contain a pattern due to errors in the measured heights or gravity data and many other types of information, which are used in geoid modelling process. In any case, when we discuss about error, we mean uncertainty or random error rather than any systematic bias. Therefore, it is important to model any systematic trend and remove it from $w$. Here, we present how this process is done theoretically. Let the following Gauss-Helmert adjustment model

$$
\mathbf{A x}+\mathbf{B \epsilon}=0-\mathbf{B L}=\mathbf{w}
$$

with

$$
\mathrm{E}\left\{\boldsymbol{\epsilon} \boldsymbol{\epsilon}^{\mathrm{T}}\right\}=\sigma^{2} \mathbf{Q}+\sigma_{N}^{2} \mathbf{Q}_{N}
$$

where $\mathbf{B}=\left[\begin{array}{ll}\mathbf{I} & -\mathbf{I}\end{array}\right]$ with $\mathbf{I}$ as an identity matrix with size of $n$ the total number of data, $\mathbf{L}=\left[\begin{array}{ll}\mathbf{h}-\mathbf{H} & \mathbf{N}\end{array}\right]^{\mathrm{T}}$ is the observation vector containing vector of the differences between ellipsoidal and orthometric heights, $h$ - $H$ and the geoid height $N, \boldsymbol{\epsilon}$ is the random error in the data with $\mathrm{E}\{\boldsymbol{\epsilon}\}=0$. The matrix $\mathbf{A}$ is related to the mathematical model, which is used for modelling the biases or systematic behaviour of $w$. There are well-known 4-, 5- and 7-parameter models for absorbing the shift and tilts of $w$ (e.g. Fotopoulos 2005, Eshagh 2010):

$$
\begin{gathered}
f_{4}(\varphi, \lambda)=x_{0}+x_{1} \cos \varphi \cos \lambda+x_{2} \cos \varphi \sin \lambda+x_{3} \sin \varphi \\
f_{5}(\varphi, \lambda)=x_{0}+x_{1} \cos \varphi \cos \lambda+x_{2} \cos \varphi \sin \lambda+x_{3} \sin \varphi+x_{4} \sin ^{2} \varphi
\end{gathered}
$$

$f_{7}(\varphi, \lambda)=x_{0}+x_{1} \cos \varphi \cos \lambda+x_{2} \cos \varphi \sin \lambda+x_{3} \sin \varphi+x_{4} k^{-1} \cos \varphi \sin \varphi \cos \lambda++x_{5} k^{-1} \cos \varphi \sin \varphi \sin \lambda+x_{6} k^{-1} \sin ^{2} \varphi$

where $\varphi$ and $\lambda$ are, respectively, the latitude and longitude of each point having the GL and geoid heights, $x_{i}, i=0,1, \ldots$ stands for the regression coefficients of each model absorbing the shifts and tilts of $w$, and $k=\left(1-e^{2} \sin ^{2} \varphi\right)^{\frac{1}{2}}, e^{2}$ is the reference ellipsoid's eccentricity. The first term of Eq. (12) can be written in matrix forms, based on the number of parameters which are used to model the trends. For 4-, 5- and 7-parameter corrective surfaces, they are, respectively

$$
\begin{gathered}
\mathbf{A x}=\left[\begin{array}{cccc}
1 & \cos \varphi_{1} \cos \lambda_{1} & \cos \varphi_{1} \sin \lambda_{1} & \sin \varphi_{1} \\
1 & \cos \varphi_{2} \cos \lambda_{2} & \cos \varphi_{2} \sin \lambda_{2} & \sin \varphi_{2} \\
\vdots & \vdots & \vdots & \vdots \\
1 & \cos \varphi_{n} \cos \lambda_{n} & \cos \varphi_{n} \sin \lambda_{n} & \sin \varphi_{n}
\end{array}\right]\left[\begin{array}{c}
x_{0} \\
x_{1} \\
x_{2} \\
x_{3}
\end{array}\right] \\
\mathbf{A x}=\left[\begin{array}{ccccc}
1 & \cos \varphi_{1} \cos \lambda_{1} & \cos \varphi_{1} \sin \lambda_{1} & \sin \varphi_{1} & \sin ^{2} \varphi_{1} \\
1 & \cos \varphi_{2} \cos \lambda_{2} & \cos \varphi_{2} \sin \lambda_{2} & \sin \varphi_{2} & \sin ^{2} \varphi_{2} \\
\vdots & \vdots & \vdots & \vdots & \vdots \\
1 & \cos \varphi_{n} \cos \lambda_{n} & \cos \varphi_{n} \sin \lambda_{n} & \sin \varphi_{n} & \sin ^{2} \varphi_{n}
\end{array}\right]\left[\begin{array}{c}
x_{0} \\
x_{1} \\
x_{2} \\
x_{3} \\
x_{4}
\end{array}\right]
\end{gathered}
$$

$\mathbf{A x}=\left[\begin{array}{ccccccc}1 & \cos \varphi_{1} \cos \lambda_{1} & \cos \varphi_{1} \sin \lambda_{1} & \sin \varphi_{1} & \cos \varphi_{1} \sin \varphi_{1} \cos \lambda_{1} / k_{1} & \cos \varphi_{1} \sin \varphi_{1} \sin \lambda_{1} / k_{1} & \sin ^{2} \varphi_{1} / k_{1} \\ 1 & \cos \varphi_{2} \cos \lambda_{2} & \cos \varphi_{2} \sin \lambda_{2} & \sin \varphi_{2} & \cos \varphi_{2} \sin \varphi_{2} \cos \lambda_{2} / k_{2} & \cos \varphi_{2} \sin \varphi_{2} \sin \lambda_{2} / k_{2} & \sin ^{2} \varphi_{2} / k_{2} \\ \vdots & \vdots & \vdots & \vdots & \vdots & \vdots & \vdots \\ 1 & \cos \varphi_{n} \cos \lambda_{n} & \cos \varphi_{n} \sin \lambda_{n} & \sin \varphi_{n} & \cos \varphi_{n} \sin \varphi_{n} \cos \lambda_{n} / k_{n} & \cos \varphi_{n} \sin \varphi_{n} \sin \lambda_{n} / k_{n} & \sin ^{2} \varphi_{n} / k_{n}\end{array}\right]\left[\begin{array}{c}x_{0} \\ x_{1} \\ x_{2} \\ x_{3} \\ x_{4} \\ x_{5} \\ x_{6}\end{array}\right]$ 
The least-squares solution of Eq. (12) is:

$$
\hat{\mathbf{x}}=\left(\mathbf{A}^{\mathrm{T}}\left(\sigma^{2} \mathbf{Q}+\sigma_{N}^{2} \mathbf{Q}_{N}\right)^{-1} \mathbf{A}\right)^{-1} \mathbf{A}^{\mathrm{T}}\left(\sigma^{2} \mathbf{Q}+\sigma_{N}^{2} \mathbf{Q}_{N}\right)^{-1} \mathbf{w}
$$

with the variance-covariance matrix of the estimates:

$$
\hat{\mathbf{C}}_{\hat{\mathbf{x}}}=\hat{\sigma}_{0}^{2}\left(\mathbf{A}^{\mathrm{T}}\left(\sigma^{2} \mathbf{Q}+\sigma_{N}^{2} \mathbf{Q}_{N}\right)^{-1} \mathbf{A}\right)^{-1}
$$

where $\hat{\sigma}_{0}^{2}$ is the a posteriori variance factor with the following formula:

$$
\hat{\sigma}_{0}^{2}=\frac{\hat{\boldsymbol{\epsilon}}^{\mathrm{T}}\left(\sigma^{2} \mathbf{Q}+\sigma_{N}^{2} \mathbf{Q}_{N}\right)^{-1} \hat{\boldsymbol{\epsilon}}}{n-m}
$$

with

$$
\hat{\boldsymbol{\epsilon}}=\mathbf{w}-\mathbf{A} \hat{\mathbf{x}}
$$

and $m$ is the number of unknown parameters. The estimated a posteriori variance factor should be in the following CI:

$$
\frac{\sigma_{0}^{2} \chi_{\alpha / 2, n-m}^{2}}{n-m} \leq \hat{\sigma}_{0}^{2} \leq \frac{\sigma_{0}^{2} \chi_{1-\alpha / 2, n-m}^{2}}{n-m}
$$

where $\sigma_{0}^{2}$ is a priori variance factor, which we assumed that it is equal to $1 . \chi_{\alpha / 2, n-m}^{2}$ and $\chi_{1-\alpha / 2, n-m}^{2}$ are the values, selected from chi-square distribution based on the significant level of $\alpha$ and the degree of freedom $n-m$. $\hat{\sigma}_{0}^{2}$ will be in the interval (22) if there is no gross error on the data, the mathematical model presents the data properly and the a priori error of the data is in agreement with the size of residuals.

To test significance of the estimated parameters, the following interval should be constructed for each estimated coefficient of the model:

$$
-\tau_{\alpha / 2, n-m} \leq \frac{\hat{x}_{i}}{\hat{\sigma}_{\hat{x}_{i}}} \leq \tau_{\alpha / 2, n-m}, x_{i}, i=0,1, \ldots
$$

where $\tau_{\alpha / 2, n-m}$ is the value, which is selected from $\tau$ distribution with the significance level of $\alpha$ and the degree of freedom $n-m$. $\tau$-distribution has the following relation with t-distribution (Pope 1976):

$$
\tau_{\alpha / 2, n-m}=\frac{t_{\alpha / 2, n-m-1} \sqrt{n-m}}{\sqrt{n-m+t_{\alpha / 2, n-m-1}^{2}}} .
$$

If the ratio of value estimated parameter and its error fall in this interval, then that parameter is not significantly different from 0 and can be removed from the model.

\section{Regional estimation of variance of the geoid model}

Under the assumption that errors of GL data are realistic, and the mathematical model of the corrective surface presents the systematic trends and removes them properly, we can, according to Eq. (21) and Eq. (22), write:

$$
\sigma_{N}^{2} \sigma_{0}^{2} \chi_{\alpha / 2, n-m}^{2} \leq \hat{\boldsymbol{\epsilon}}^{\mathrm{T}}\left(\sigma^{2} / \sigma_{N}^{2} \mathbf{Q}+\mathbf{Q}_{N}\right)^{-1} \hat{\boldsymbol{\epsilon}} \leq \sigma_{N}^{2} \sigma_{0}^{2} \chi_{1-\alpha / 2, n-m}^{2} .
$$

For solving Eq. (25), the upper and lower bounds of the interval are considered separately. When the lower bound is consider we obtain the following iterative estimators of variance for the geoid model:

$$
\left(\sigma_{N}^{2}\right)^{k+1} \leq \frac{\hat{\boldsymbol{\epsilon}}^{\mathrm{T}}\left(\sigma^{2} /\left(\sigma_{N}^{2}\right)^{k} \mathbf{Q}+\mathbf{Q}_{N}\right)^{-1} \hat{\boldsymbol{\epsilon}}}{\sigma_{0}^{2} \chi_{\alpha / 2, n-m}^{2}}
$$

and when the upper bound

$$
\left(\sigma_{N}^{2}\right)^{k+1} \geq \frac{\hat{\boldsymbol{\epsilon}}^{\mathrm{T}}\left(\sigma^{2} /\left(\sigma_{N}^{2}\right)^{k} \mathbf{Q}+\mathbf{Q}_{N}\right)^{-1} \hat{\mathbf{\epsilon}}}{\sigma_{0}^{2} \chi_{1-\alpha / 2, n-m}^{2}} .
$$

where $k$ stands for the iteration number.

In this case, two values are obtained based on the degree of freedom and the selected significant level for the lower and upper bound of the geoid height variance.

\section{Local estimation of variance of the geoid model}

The iterative variance estimators (26) and (27) are of regional type, meaning that they give estimates of variance over a region, in this study, each country. Therefore, it is somehow an average estimate of the upper and lower bounds for the geoid error. Another way for estimating the variance for the geoid heights is to consider $w$ at each point separately after detrending. In this case, we can estimate the variance for each point individually. To do so, we use the standardised residuals, meaning that the ratio of each residual after detredning and its estimated error should have normal distribution with a significant level $\alpha$ :

$$
\left|\frac{\hat{\varepsilon}_{i}}{\sigma_{\hat{\varepsilon}_{i}}}\right| \leq Z_{\frac{\alpha}{2}} .
$$

As shown in Eq. (21), the residuals are:

$$
\hat{\boldsymbol{\epsilon}}=\mathbf{W}-\mathbf{A} \hat{\mathbf{x}}=\mathbf{R} \mathbf{W}
$$

where

$$
\begin{aligned}
& \mathbf{R}= \\
& \left(\mathbf{I}-\left(\mathbf{A}^{\mathrm{T}}\left(\sigma^{2} / \sigma_{N}^{2} \mathbf{Q}+\mathbf{Q}_{N}\right)^{-1} \mathbf{A}\right)^{-1} \mathbf{A}^{\mathrm{T}}\left(\sigma^{2} / \sigma_{N}^{2} \mathbf{Q}+\mathbf{Q}_{N}\right)^{-1}\right) .
\end{aligned}
$$


By applying the error propagation law to Eq. (29), the variance-covariance matrix of the estimated residuals is obtained:

$$
\mathbf{C}_{\hat{\mathbf{e}}}=\mathbf{R C}_{\mathbf{w}} \mathbf{R}^{\mathrm{T}}
$$

where

$$
\mathbf{C}_{\mathbf{w}}=\sigma^{2} \mathbf{Q}+\sigma_{N}^{2} \mathbf{Q}_{N}
$$

Now, we square both hand sides of Eq. (28) and ignore the absolute value as a squared value is always positive:

$$
\frac{\hat{\varepsilon}_{i}^{2}}{\sigma_{\hat{\varepsilon}_{i}}^{2}} \leq Z_{\frac{\alpha}{2}}^{2} .
$$

After simplification of Eq. (29)-(31) and substituting the results into Eq. (32) we obtain:

$$
\frac{\mathbf{r}_{i} \mathbf{w} \mathbf{r}_{i} \mathbf{w}}{\sigma_{N}^{2} \mathbf{r}_{i}\left(\sigma^{2} / \sigma_{N}^{2} \mathbf{Q}+\mathbf{Q}_{N}\right) \mathbf{r}_{i}^{\mathrm{T}}} \leq Z_{\frac{\alpha}{2}}^{2}
$$

where $\mathbf{r}_{i}$ is the $i$-th row of the matrix R. Eq. (33) can be written as:

$$
\left(\sigma_{N}^{2}\right)^{k+1} \geq \frac{\left(\mathbf{r}_{i}\right)^{k} \mathbf{w}\left(\mathbf{r}_{i}\right)^{k} \mathbf{w}}{Z_{\frac{\alpha}{2}}^{2}\left(\mathbf{r}_{i}\right)^{k}\left(\sigma^{2} /\left(\sigma_{N}^{2}\right)^{k} \mathbf{Q}+\mathbf{Q}_{N}\right)\left(\mathbf{r}_{i}^{\mathrm{T}}\right)^{k}}
$$

which is in fact an iterative estimator of the variance for the geoid height. This estimator is local meaning that it is applied to each point. The iteration process is repeated until the estimate converges and does not change by further iteration.

\section{Application}

In this section, the data over the Nordic countries are presented. Later different statistical tests as well as detrending model will be applied on $w$. A CI will be estimated for the geoid error over each country and the local variance estimator will be applied at the GL points.

\subsection{The NKG2015 geoid model and GNSS/levelling data}

The NKG2015 geoid model has a limited coverage between the latitudes $53^{\circ} \mathrm{N}$ and $73^{\circ} \mathrm{N}$ the longitudes $0^{\circ} \mathrm{E}$ and $34^{\circ} \mathrm{E}$ containing Sweden, Norway, Denmark and Finland. Since the GL data are collected only over lands, our study area will be limited to lands as well. The NKG2015 geoid model is shown in the background of the GL points over the study area in Fig. 1a. The lowest value of the geoid heights is seen in the southern of Finland and reaches to 15 metres and the highest value in the mountainous area of Norway to 50 metres.

The GL data contain ellipsoidal heights measured by GNSS receivers and orthometric heights. As was mentioned before, the difference between these two heights gives us the geoid height theoretically. In other words, the GL data can be used as check points for quality test of the NKG2015 geoid model. Note that not all the GL data are approved for this purpose by the national responsible authorities of the Nordic country, even if they are rather good. 197 GL points amongst all have been selected for Sweden, and 697, 923 and 50, respectively, for Denmark, Norway and Finland. The estimated precisions for these data are $11 \mathrm{~mm}$ for 25 points and $14 \mathrm{~mm}$ for the rest of the points in Sweden, 10, 15 and 25, respectively, for Denmark, Norway and Finland.

According to Fig. 1a the GL points over Finland have a rather sparse distribution and the geoid model is smooth there. The densest distribution of the GL points is seen in Denmark, a flat and smaller area comparing to the rest of the countries. In this area the geoid cannot fluctuate significantly. The GL points have evenly covered Sweden, but in mountainous Norway establishing these points with such a uniform distribution is not possible.

The map of differences between the NKG2015 geoid model and the GL data, or discrepancies $w$, are presented in Fig. 1b. These differences is gridded for a better visualisation purpose in this figure. As seen, they are almost positive meaning that there should be a shift between the geoid model and the GL data and smaller in the southern parts of Sweden and Norway, and rather constant over Denmark and Finland. Large differences, reaching to $20 \mathrm{~cm}$, are observed along the Norwegian coastal line, north of Sweden and Finland.

\subsubsection{Test of discrepancies}

As Eq. (4) shows, the discrepancies play an important role for determining the quality of the geoid heights. They should be normally distributed, but this is not the case in practice, as there may exist blunders amongst them and/or they may contain some systematic patterns or trends. Here, our goal is to test normality of them by filtering based on the confidence intervals (CIs) of $95 \%$ and $99.7 \%$, the chisquare goodness-of-fit (GOF) test and test of skewness and kurtosis of the histograms of $w$. 


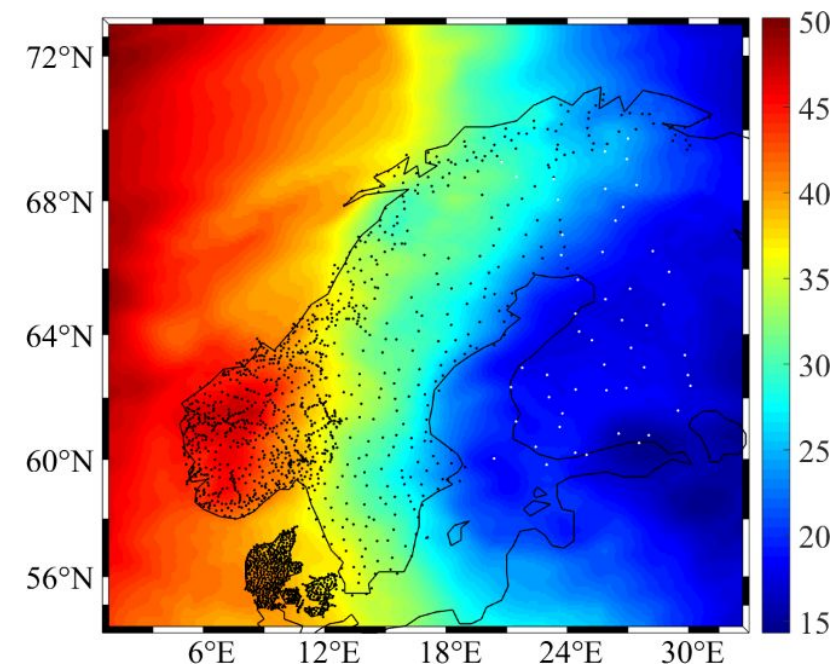

(a)

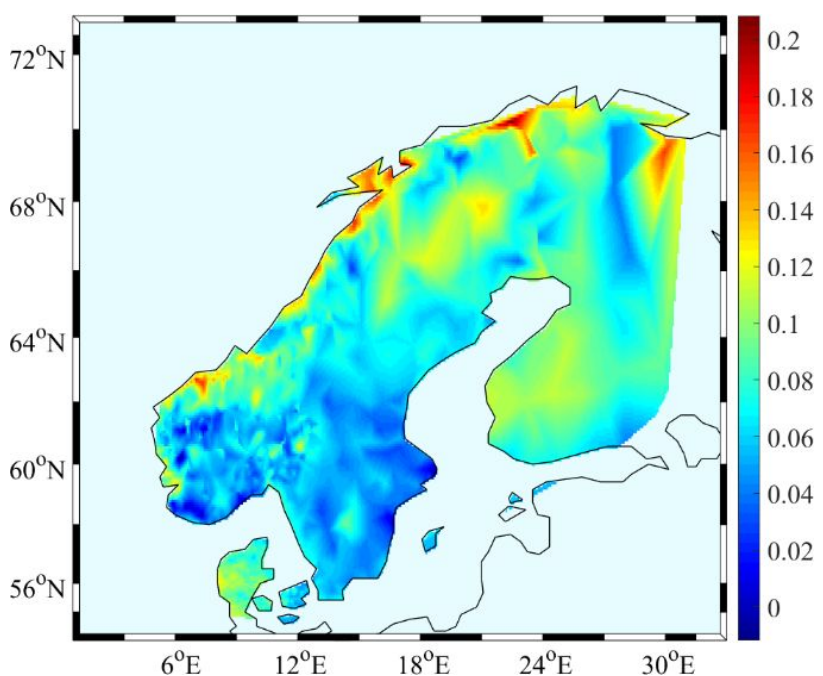

(b)

Fig. 1. a) Distribution of GL points over the Nordic countries on the background of NKG2015 geoid model, b) differences between the geoid heights and GL data (discrepancies) [m]

\subsubsection{Filtering the discrepancies based on confidence intervals of $95 \%$ and $99.7 \%$}

Here, we filter $w$ according to the CIs of $95 \%$ and $99.7 \%$ and after that we check to see possible improvements of their distribution according to the normal standard distribution for the significant level $\alpha=0.01$. Therefore, it is rather simple to construct the CIs according to Eq. (6), from the mean value and standard deviation of the discrepancies.

Figure 2a shows the removed GL points after filtering the discrepancies based on the CI of $95 \%$ and Fig. $2 \mathrm{~b}$ a similar map based on $99.7 \%$. The deleted points are located close to coastal lines and over the mountainous areas. In the case of considering the $\mathrm{CI}$ of $99.7 \%$ one point in

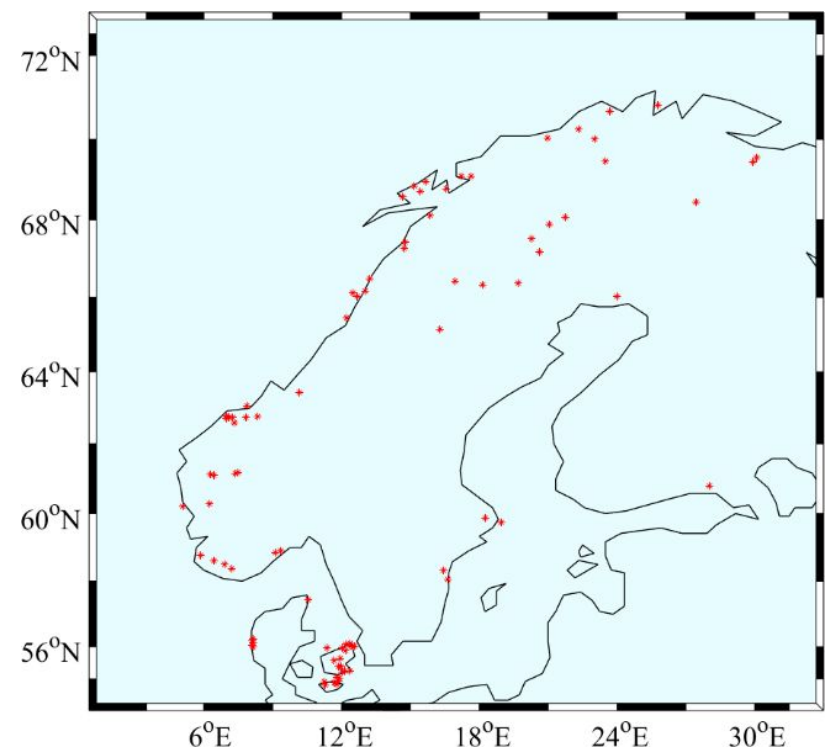

(a)

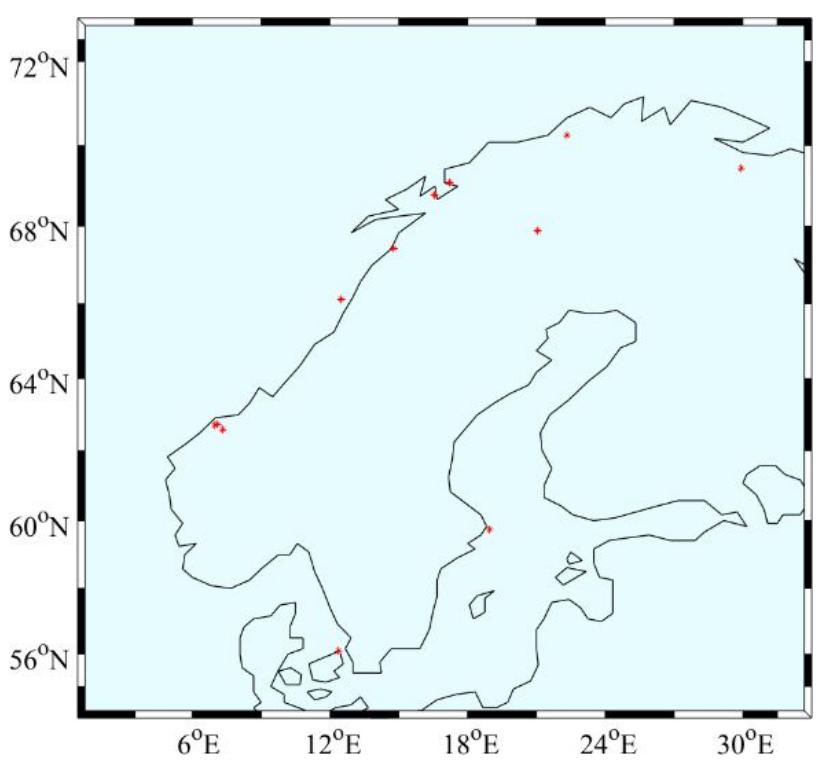

(b)

Fig. 2. a) GL outliers from filtering with a confidence interval of a) $95 \%$ and b) $99.7 \%$.

Denmark and one in Finland were deleted and two in the northern and mountainous part of Sweden and few points close to the coastal lines in Norway. Heterogeneous quality of the topographic heights and gravimetry measurements in geoid computation on the one hand and other errors in measuring the normal and geodetic heights by GNSS receivers on the other hand are the probable reasons causing that some of the discrepancies fall outside the CIs. 


\subsubsection{Chi-square goodness-of-test}

As described in Section 3.2, the chi-square GOF test is passed if the $y$ value is smaller than $\chi_{\alpha, n b-1}^{2}$, this would indicate a good fit to the normal distribution curve. This test has been applied to the unfiltered and filtered discrepancies and its results are summarised in Table 1. As observed, only Sweden and Finland discrepancies pass this test before and after filtering them, therefore probably they have normal distributions. Norway and Denmark's discrepancies clearly fail the test, and it cannot be said that they are normally distributed. What we can conclude here is that the filtering process does not necessarily improve the distribution of the discrepancies.

Table 1. Chi-square goodness-of-fit test of normality before and after filtering based on two confidence intervals of $95 \%$ and $99.7 \%$, $\mathrm{P}$ and $\mathrm{F}$ stand for pass and fail, respectively [unitless]

\begin{tabular}{|l|c|c|c|c|c|c|c|c|c|}
\hline \multirow{2}{*}{ Region } & \multicolumn{3}{|c|}{ Before filtering } & \multicolumn{3}{|c|}{ Filtering 95\% CI } & \multicolumn{3}{|c|}{ Filtering 99.7\% Cl } \\
\cline { 2 - 11 } & $y$ & $\chi_{\alpha, n b-1}^{2}$ & Test & $y$ & $\chi_{\alpha, n b-1}^{2}$ & Test & $y$ & $\chi_{\alpha, n b-1}^{2}$ & Test \\
\hline Sweden & 2.3177 & 2.7955 & $\mathbf{P}$ & 2.7565 & 3.5485 & $\mathbf{P}$ & 3.1471 & 3.3272 & $\mathbf{P}$ \\
\hline Denmark & 6.4827 & 3.5485 & $\mathbf{F}$ & 5.6961 & 3.7506 & $\mathbf{F}$ & 5.7536 & 3.7506 & $\mathbf{F}$ \\
\hline Norway & 8.1113 & 3.3272 & $\mathbf{F}$ & 8.1400 & 3.7506 & $\mathbf{F}$ & 7.6237 & 3.5485 & $\mathbf{F}$ \\
\hline Finland & 2.1696 & 3.0802 & $\mathbf{P}$ & 2.7350 & 2.7955 & $\mathbf{P}$ & 2.1696 & 3.0802 & $\mathbf{P}$ \\
\hline
\end{tabular}

\subsubsection{Test of skewness and kurtosis}

A complementary way to test the normality of the discrepancies is to check skewness and kurtosis of their histogram. Table 2 shows the standardised skewness and kurtosis, which can be derived by dividing each one by respective error; see Section 3.3. Based on a significant level of 0.05 of the normal standard distribution these ratios should have a value between -1.96 and 1.96. Table 2 that the kurtosis before filtering is 3.98, significantly different from 0 , meaning that the histogram is tailed significantly. After filtering, this ratio will be 2.08 for CI of $95 \%$ and 1.44 for $99.7 \%$, which works rather fine but makes the histogram slightly skewed. In Denmark, the histogram of the filtered discrepancies by $95 \% \mathrm{CI}$ is closer to normal distribution. In Norway, this histogram is far from a normal distribution even after filtering by $95 \%$ and $99.7 \%$ CIs, the standardised kurtosis will be smaller than 1.96, but the distribution is still skewed. However, as the table shows the standardised skewness is smaller for filtering based on 95\% CI and probability will be smaller than 1.96 if they discrepancies are filtered by smaller percentage of CI. The his- togram is very close to the normal distribution in Finland even without applying filters.

Table 2. The standardised skewness and kurtosis values before and after filtering of the discrepancies based on $\mathrm{Cls}$ of $95 \%$ and $99.7 \%$ [unitless]

\begin{tabular}{|c|c|c|c|c|c|c|}
\hline & \multicolumn{2}{|c|}{ No filtering } & \multicolumn{2}{c|}{ Filtering 95\% $\mathrm{Cl}$} & \multicolumn{2}{c|}{ Filtering 99\% Cl } \\
\hline Region & $S / \sigma_{S}$ & $K / \sigma_{K}$ & $S / \sigma_{S}$ & $K / \sigma_{K}$ & $S / \sigma_{S}$ & $K / \sigma_{K}$ \\
\hline Sweden & 1.54 & 3.98 & 0.38 & 2.08 & 2.29 & 1.44 \\
\hline Denmark & -3.56 & -1.38 & -2.45 & -2.81 & +3.33 & +1.63 \\
\hline Norway & 9.08 & 6.13 & 4.74 & -1.87 & 5.93 & 1.98 \\
\hline Finland & -1.18 & -1.28 & -1.04 & -1.27 & -1.18 & +1.28 \\
\hline
\end{tabular}

\subsubsection{Least-squares detrending by the corrective surfaces}

Now, the presented 4-, 5- and 7-parameters corrective surfaces in Eqs. (13)-(15), are applied for detrending the discrepancies. However, the main question is which one of these three models has the best fit to the discrepancies of which country? To answer this question, we perform some statistical tests on the results of detrending process. The first test is to check the significance of each estimated parameter to find how many of the selected parameters in the corrective surface models have significant role in detrending. The test statistic has been already presented in Eq. (26). This means that the ratio of the estimated parameter and its estimated error should have $\tau$-distribution with significant level of and degree of freedom of $n-m$. Note that $\tau$-, $\mathrm{t}$ - and normal distribution will be very close to each other when the degree of freedom is large, like this study. Proper selection of the corrective surface is important as by underestimating the number of the parameters, some parts of the trends, which are, in fact, systematic patterns will contribute to the random errors and by overestimating the parameters some parts of the random noise will be absorbed in the trend. Therefore, checking the normality of the residuals after detrending will be very helpful. If they have normal distribution, probably the selected model has the best fit to that area.

For selecting the best corrective surface, no error is considered for the geoid and GL data, as their improper weights can influence the fitting. This means that in the mathematical model for the least-squares estimation of the parameters and their variance-covariance matrix, we 
consider $\sigma^{2}=\sigma_{N}^{2}=1$ and $\mathbf{Q}=\mathbf{Q}_{\mathbf{N}}=\mathbf{I}$, in Eqs. (19), (20) and (21).

The detrending will be performed in three different scenarios for each model, detrending the discrepancies without any filtering, filtered by CIs of $95 \%$ and $99.7 \%$. In fact, by considering the filtered discrepancies, we have reduced the risk of presence of outliers in the least-squares detrending procedure.

Table 3 summarises the ratio of the estimated parameters and their errors for each corrective surface model before and after filtering of discrepancies with CIs of $95 \%$ and $99.7 \%$. The significant ratios are highlighted in the table.

As we observe, in Sweden the least-squares detrending of the nonfiltered discrepancies with 4-parameter model leads to significant values for the estimated parameters, whilst when the 5-parameter model is chosen, only one parameter comes out significant, and when 7parameter model is selected two will be statistically significant. In the case where the filtered discrepancies are used, the 4-parameter model has still significant parameters. In Denmark, the results of the significant test of the estimated parameters from detrended discrepancies before and after filtering with CI of $99.7 \%$ are similar, but when the filtering is done with a CI of 95\% some parameters of 5- and 7-parameter models comes out significant. In Norway, the test shows that the 4-parameter model has some insignificant parameters, but when 5- and 7-parameter models are applied the estimated parameters will be significant before or after filtering of discrepancies. Filtering has no significant impact on the result of the significant test of the estimated parameters and in all cases of using the 4- and 7parameter models in Finland.

Looking at the significance of the estimated parameter can somehow give us some idea about the quality of the fitting or detrending process. However, it is not easy to conclude about the results rigorously. Therefore, more statistical tests are required for doing so. One method for testing the fit of a mathematical model to data is to test the normality of residuals. If the residuals after detrending are normality distributed, we may conclude that the fitting and detrending process have been successful. Here, a chi-square GOF test of the residuals to the normal distribution with the same mean and standard deviation of them is needed.

Table 4 represents the results of the chi-square GOF test of the residuals estimated from least-squares detrending using 4-, 5- and 7-parameter models. It shows that this test is passed only in the case of detrending the filtered discrepancies with a CI of $95 \%$ in Sweden. The importance of filtering discrepancies over this area is clear. All three models passed the test when the filtered discrepancies with a
Table 3. Standardised estimated parameters from least-squares detrending process with discrepancies, before and after filtering the discrepancies by $\mathrm{Cls}$ of $95 \%$ and $99.7 \%$, column 4,5 and 7 represents the number of parameters in each corrective surface model [unitless]

\begin{tabular}{|c|c|c|c|c|c|c|c|c|c|}
\hline \multirow[t]{2}{*}{ Region } & \multicolumn{3}{|c|}{ No filtering } & \multicolumn{3}{|c|}{ Filtering 95\% Cl } & \multicolumn{3}{|c|}{ Filtering 99.7\% Cl } \\
\hline & 4 & 5 & 7 & 4 & 5 & 7 & 4 & 5 & 7 \\
\hline \multirow{7}{*}{ 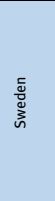 } & 7.8042 & 2.4988 & -0.2906 & 5.7222 & 1.6996 & -3.0560 & 7.3781 & 2.2921 & -1.2274 \\
\hline & -8.1859 & -1.0437 & -3.0213 & -5.9264 & -0.9919 & -4.8387 & -7.7442 & -1.1011 & -3.4870 \\
\hline & -8.2632 & -1.7550 & -1.2674 & -6.0110 & -1.5227 & -3.8083 & -7.6515 & -1.7357 & -2.0918 \\
\hline & -7.5115 & -1.1156 & 0.4457 & -5.4909 & -0.6371 & 3.2151 & -7.0944 & -0.9654 & 1.3699 \\
\hline & & 0.7039 & 3.2343 & & 0.3245 & 4.6896 & & 0.5716 & 3.5192 \\
\hline & & & 1.5990 & & & 3.9570 & & & 2.3391 \\
\hline & & & 0.9785 & & & 3.6213 & & & 1.8551 \\
\hline \multirow{7}{*}{$\begin{array}{l}\text { L } \\
\text { E } \\
\text { Ĕ } \\
\text { o }\end{array}$} & -6.7438 & -6.2453 & -1.3205 & -6.5838 & -6.4575 & -1.4712 & -6.7380 & -6.3720 & -1.3666 \\
\hline & 6.9390 & 3.4510 & -1.8351 & 6.7766 & 2.6910 & -2.1807 & 6.9323 & 3.2658 & -1.9184 \\
\hline & 3.7567 & 1.4148 & -1.3021 & 3.7794 & 0.8613 & -1.4164 & 3.7589 & 1.2445 & -1.3398 \\
\hline & 6.7275 & 2.9153 & 1.3424 & 6.5670 & 3.3978 & 1.4726 & 6.7221 & 3.1220 & 1.3840 \\
\hline & & -1.4673 & 1.8337 & & -2.0544 & 2.1623 & & -1.6859 & 1.9132 \\
\hline & & & 1.2991 & & & 1.3980 & & & 1.3334 \\
\hline & & & 1.2976 & & & 1.4289 & & & 1.3391 \\
\hline \multirow{7}{*}{$\frac{\sqrt{\frac{\pi}{2}}}{2}$} & -1.6173 & -2.5840 & -5.4335 & -1.9726 & -4.1905 & -5.3001 & -2.04696 & -3.0131 & -5.2428 \\
\hline & -0.1883 & -2.1983 & 4.6887 & 0.3860 & -3.4355 & 2.2192 & 0.29016 & -2.3421 & 3.9081 \\
\hline & -5.0293 & -5.2702 & -4.3155 & -4.8489 & -6.2238 & -4.3529 & -4.67080 & -5.2454 & -4.2117 \\
\hline & 2.4018 & 2.4662 & 5.0856 & 2.7233 & 4.0528 & 5.0609 & 2.82515 & 2.8489 & 4.9411 \\
\hline & & -2.3266 & -5.2035 & & -3.9029 & -2.7724 & & -2.6873 & -4.4223 \\
\hline & & & 3.9040 & & & 4.0114 & & & 3.8345 \\
\hline & & & 4.6978 & & & 4.6588 & & & 4.5581 \\
\hline \multirow{7}{*}{ 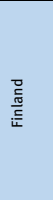 } & -3.4636 & -2.4163 & 4.2145 & -3.0100 & -2.18458 & 4.3613 & -3.4636 & -2.4163 & 4.2145 \\
\hline & 3.6530 & -1.0278 & 4.1686 & 3.1675 & -0.95731 & 4.3093 & 3.6530 & -1.0278 & 4.1686 \\
\hline & 2.6167 & -1.3050 & 4.7270 & 2.3292 & -1.19963 & 4.8317 & 2.6167 & -1.3050 & 4.7270 \\
\hline & 3.4980 & 2.0371 & -4.3214 & 3.0460 & 1.85356 & -4.4623 & 3.4980 & 2.0371 & -4.3214 \\
\hline & & -1.8761 & -4.3168 & & -1.71154 & -4.4490 & & -1.8761 & -4.3168 \\
\hline & & & -4.7693 & & & -4.8623 & & & -4.7693 \\
\hline & & & -4.6595 & & & -4.7746 & & & -4.6595 \\
\hline
\end{tabular}

CI of $99.7 \%$ is applied in detrending for Denmark. In Norway, the test is passed only when a 7-paramter model is applied for the filtered data with CI of 95\%. In Finland, it seems that that filtering of discrepancies has no role.

Table 4. Chi-square GOF test of the estimated residuals before and after least-squares detrending of filtered and nonfiltered discrepancies [unitless]

\begin{tabular}{|c|c|c|c|c|c|c|c|c|c|c|}
\hline & \multirow[t]{2}{*}{$P$} & \multicolumn{3}{|c|}{ Before filtering } & \multicolumn{3}{|c|}{ Filtering $95 \% \mathrm{Cl}$} & \multicolumn{3}{|c|}{ Filtering $99.7 \% \mathrm{Cl}$} \\
\hline & & $\mathrm{Y}$ & $x$ & Test & $\mathrm{Y}$ & $\mathrm{x}$ & Test & $\mathrm{Y}$ & $\mathrm{x}$ & Test \\
\hline & 4 & 3.4956 & 2.7955 & $\mathrm{~F}$ & 1.8522 & 2.7955 & $\mathbf{P}$ & 3.3494 & 2.7955 & $\mathbf{F}$ \\
\hline & 5 & 3.4659 & 2.7955 & $\mathrm{~F}$ & 1.5545 & 2.7955 & $\mathbf{P}$ & 3.5051 & 2.7955 & $\mathbf{F}$ \\
\hline & 7 & 3.4659 & 2.7955 & $\mathrm{~F}$ & 2.0391 & 2.7955 & $P$ & 3.2553 & 3.0802 & $F$ \\
\hline & 4 & 2.2803 & 3.3272 & $\mathbf{P}$ & 1.4384 & 3.3272 & $\mathbf{p}$ & 2.3299 & 3.3272 & $\mathrm{P}$ \\
\hline & 5 & 2.2776 & 3.3272 & $\mathbf{P}$ & 1.2419 & 3.3272 & $\mathrm{P}$ & 2.2042 & 3.3272 & $\mathbf{P}$ \\
\hline & 7 & 4.7151 & 3.3272 & $\mathrm{~F}$ & 3.8108 & 3.3272 & $\mathrm{~F}$ & 2.8612 & 3.0802 & P \\
\hline & 4 & 5.6198 & 3.3272 & $\bar{F}$ & 5.1856 & 3.5485 & $\bar{F}$ & 5.8192 & 3.5485 & $\bar{F}$ \\
\hline है & 5 & 5.4074 & 3.0802 & $\mathrm{~F}$ & 4.8741 & 3.5485 & $\mathrm{~F}$ & 5.7213 & 3.5485 & $\mathrm{~F}$ \\
\hline & 7 & 5.2334 & 3.3272 & $\mathrm{~F}$ & 3.1235 & 3.5485 & $P$ & 5.2534 & 3.5485 & $F$ \\
\hline & 4 & 2.1996 & 3.0802 & $\bar{p}$ & 1.7919 & 2.7955 & $\bar{p}$ & 2.1996 & 3.0802 & 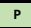 \\
\hline 들 & 5 & 2.8365 & 3.0802 & $\mathbf{P}$ & 2.0866 & 2.7955 & $\mathbf{P}$ & 2.8365 & 3.0802 & $\mathbf{P}$ \\
\hline & 7 & 1.7144 & 3.0802 & $\mathbf{p}$ & 1.6809 & 3.0802 & $\mathbf{P}$ & 1.7144 & 3.0802 & $\mathbf{P}$ \\
\hline
\end{tabular}

In Table 5, we present the results of the skewness and kurtosis significant test of the estimated residuals after detrending of the nonfiltered and filtered discrepancies by the three corrective surface models. From this ta- 
ble, we can conclude that the best fit is achieved in Sweden when the 4-parameter corrective surface is chosen for the detrending the discrepancies with $\mathrm{CI}$ of $95 \%$. The 4parameter surface in Denmark and Finland for unfiltered discrepancies and finally the 7-parameter corrective surface model for detrending of filtered discrepancies by a CI of $95 \%$.

Figure 3 is the map of residuals after detrending the filtered or non-filtered discrepancies of each country based on the selected corrective surface models. The map shows that the residuals range from $-5 \mathrm{~cm}$ to $6.3 \mathrm{~cm}$, the extreme values are seen mostly in Norway over its mountains and close to coast. However, we should admit that the topography of Norway is rather rough and complicated, even if we could select a 7-parameter model for detrending the filtered discrepancies, there is still risk that this model cannot present the trends well. These residuals and the priori errors of the GL data can be used for estimating the uncertainty of the geoid model in each country. The estimated error from these residuals are, 13, 11, 22 and $17 \mathrm{~mm}$, respectively, in Sweden, Denmark, Norway and Finland. These values are close to the claimed error for the GL heights except of Norway, which the claim is $15 \mathrm{~mm}$ and Finland 25 $\mathrm{mm}$. Simply by assuming that these claims are correct, the error of geoid will be very small. For example, in Norway, the geoid error should be $16 \mathrm{~mm}$ to match the residuals. The geoid error is not estimable based on the claimed error the GL data of Finland. In Denmark, the geoid error should be about $5 \mathrm{~mm}$.

Table 5. Values of test statistic for skewness and kurtosis of the estimated residuals based on 4-, 5- and 7-parameter trend models of discrepancies before and after filtering, significant level of the significant test is 0.05 . The column $\mathrm{P}$ shows number of parameters in each corrective surface model [unitless]

\begin{tabular}{|c|c|c|c|c|c|c|c|}
\hline & & \multicolumn{2}{|c|}{ No filtering } & \multicolumn{2}{|c|}{ Filtering $95 \% \mathrm{Cl}$} & \multicolumn{2}{|c|}{ Filtering $99.7 \% \mathrm{Cl}$} \\
\hline & $\mathrm{P}$ & $S / \sigma_{S}$ & $K / \sigma_{K}$ & $S / \sigma_{S}$ & $K / \sigma_{K}$ & $S / \sigma_{S}$ & $K / \sigma_{K}$ \\
\hline \multirow{3}{*}{ 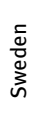 } & 4 & -3.99 & 7.28 & -2.12 & 3.61 & -2.73 & 5.67 \\
\hline & 5 & -3.95 & 7.48 & -2.13 & 3.77 & -2.74 & 5.91 \\
\hline & 7 & -1.22 & 4.73 & -0.43 & 4.33 & -0.57 & 4.21 \\
\hline \multirow{3}{*}{$\begin{array}{l}\text { 总 } \\
\sum_{\bar{\Phi}}^{ \pm}\end{array}$} & 4 & 0.77 & 1.99 & 0.53 & 1.98 & 0.81 & 2.00 \\
\hline & 5 & 0.76 & 1.94 & 0.83 & 1.96 & 0.82 & 1.92 \\
\hline & 7 & 0.89 & 3.27 & 1.41 & 3.16 & 1.05 & 3.23 \\
\hline \multirow{3}{*}{$\begin{array}{l}\text { 弯 } \\
\stackrel{0}{2}\end{array}$} & 4 & 5.10 & 6.34 & 3.22 & 1.32 & 2.65 & 2.82 \\
\hline & 5 & 5.19 & 6.39 & 3.0 & 1.03 & 2.63 & 2.72 \\
\hline & 7 & 4.58 & 6.55 & 2.89 & 1.10 & 2.31 & 2.90 \\
\hline \multirow{3}{*}{ 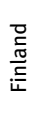 } & 4 & -1.64 & 0.20 & -2.01 & 0.56 & -1.64 & 0.20 \\
\hline & 5 & -1.52 & 0.28 & -1.93 & 0.64 & -1.52 & 0.28 \\
\hline & 7 & -0.87 & -0.75 & -1.19 & -0.36 & -0.87 & -0.75 \\
\hline
\end{tabular}

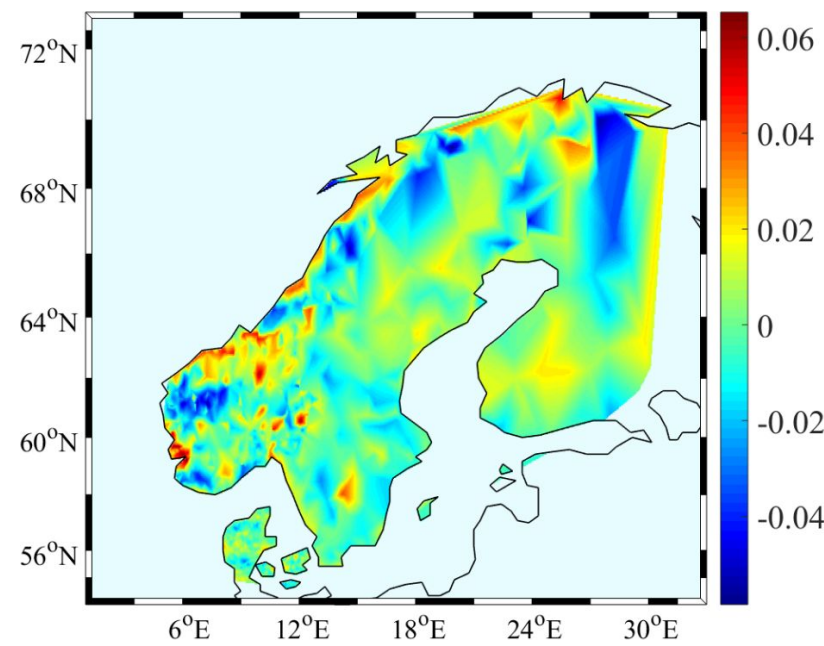

Fig. 3. Residuals after least-squares detrending in each country, based on the selected corrective surface models and the filtered discrepancies with $\mathrm{Cls}$ of $95 \%$ in Sweden and Norway and nonfiltered one in Denmark and Finland[m]

\subsubsection{Confidence interval for the geoid error in each country}

The discrepancies are also dependent on the errors of the GL data and the geoid model. The estimated errors for the GL heights and, some rough estimates of the geoid height errors are given by the developers (Ågren et al. 2016). Here, we apply our new method; see Eqs. (26) and (27) to estimate a CI for the error of the geoid model based on the estimated residuals and the priori errors of the GL heights with assumption that their errors are realistic. Our method is of iterative nature and the estimates are iterated 60 times until the estimated variance converges for the upper and lower bounds of the CIs with a significant level of 0.05 .

The estimated CIs for the errors of the geoid model is presented Table 7 . The CIs are compared to the estimated errors of the geoid to analyse whether the given errors are within the CIs.

The given geoid error for Sweden is within the error interval before filtering and after filtering with 99.7\% CI, except the case where the 7-parameter model is used. This could be expected as in the estimation of this given error no filtering has been applied to the discrepancies. When the filtered discrepancies are used, the CI becomes smaller, but the lower bound will be zero, which is not very realistic, but we can say that the geoid error over Sweden is less than $7 \mathrm{~mm}$.

The given geoid error for Denmark is larger than the upper bounds of the CIs, but as we can see for the case of 
detrening non-filtered discrepancies with the 4-parameter model, the geoid error will range between $1.8 \mathrm{~mm}$ to 5.2 $\mathrm{mm}$ in Denmark. This seems to be realistic as the area is considerably small and flat, the geoid does not vary significantly over the data. The situation will be different in Norway, the given error is smaller than the lower bounds of the CIs, if the discrepancies are not filtered, but after filtering and detredning with the 7-parameter model, it will be between 14.8-17.7 $\mathrm{mm}$. However, as the table shows, the error of geoid comes out zero, which is not realistic. This is because of the pessimistic error of the GL heights in Finland, which are not in agreement with the size residuals. In other words, the residuals are too small that the geoid error can contribute to them.

Table 6. Confidence intervals based on the confidence of $95 \%$ for the error of the NKG2015 geoid model. NOP means number of points, $P$ is the number of parameters in the used corrective surface model [mm]

\begin{tabular}{|c|c|c|c|c|c|c|c|c|c|c|}
\hline \multirow[b]{2}{*}{$\begin{array}{l}\text { 흠 } \\
\text { ơ }\end{array}$} & \multirow[b]{2}{*}{$\mathbf{P}$} & \multicolumn{3}{|c|}{ Before filtering } & \multicolumn{3}{|c|}{ Filtering $95 \% \mathrm{Cl}$} & \multicolumn{3}{|c|}{ Filtering $99.7 \% \mathrm{Cl}$} \\
\hline & & NOP & Lower & $\begin{array}{c}\text { Uppe } \\
r\end{array}$ & NOP & Lower & Upper & NOP & Lower & $\begin{array}{c}\text { Uppe } \\
r\end{array}$ \\
\hline \multirow{3}{*}{ 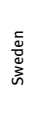 } & 4 & 19 & 3. & 11.4 & 183 & 0.0 & 6. & 195 & 3.1 & 10.1 \\
\hline & 5 & 197 & 5.4 & 11.5 & 183 & 0.0 & 6.6 & 195 & 3.1 & 10.2 \\
\hline & 7 & 197 & 4.4 & 10.8 & 183 & 0.0 & 4.6 & 195 & 1.7 & 9.4 \\
\hline \multirow{3}{*}{ 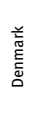 } & 4 & 67 & 1. & 5.2 & 647 & 0.0 & 3. & 674 & 1.7 & 5.2 \\
\hline & 5 & 675 & 1.8 & 5.2 & 647 & 0.0 & 3.8 & 674 & 1.7 & 5.1 \\
\hline & 7 & 675 & 1.2 & 4.9 & 647 & 0.0 & 3.0 & 674 & 1.0 & 4.7 \\
\hline \multirow{3}{*}{ 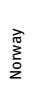 } & 4 & 902 & 20.2 & 23.2 & 858 & 15.6 & 18.5 & 893 & 18.6 & 21.4 \\
\hline & 5 & 902 & 20.1 & 23.1 & 858 & 15.4 & 18.2 & 893 & 18.6 & 21.3 \\
\hline & 7 & 902 & 19.3 & 22.2 & 858 & 14.8 & 17.7 & 893 & 17.7 & 20.6 \\
\hline \multirow{3}{*}{$\frac{D}{\frac{5}{5}}$} & 4 & 30 & 0.0 & 0.0 & 48 & 0.0 & 0.0 & 30 & 0.0 & 0.0 \\
\hline & 5 & 50 & 0.0 & 0.0 & 48 & 0.0 & 0.0 & 50 & 0.0 & 0.0 \\
\hline & 7 & 50 & 0.0 & 0.0 & 48 & 0.0 & 0.0 & 50 & 0.0 & 0.0 \\
\hline
\end{tabular}

In order to give a general idea about the error of the NKG2015 geoid model, the CIs for the error of the geoid model are determined by varying the error the GL heights from $1 \mathrm{~mm}$ to $25 \mathrm{~mm}$. In Figs. 4a-4d the graphical relations between the error of the GL heights and the error of the NKG2015 geoid model with a CI of 95\% for each country are plotted based on the best corrective surface for detrending of discrepancies in each country, and applying the method presented in Section 5. Figure 4a shows that a GL height error of $13.6 \mathrm{~mm}$ and a geoid error of $10 \mathrm{~mm}$ has a relation to the residuals that is close to the upper limit. When the error of the GL heights becomes larger than $13.6 \mathrm{~mm}$, then the error of the geoid model will come out zero, which is not logical.
Figure $4 \mathrm{~b}$ shows a similar plot but in Denmark. The $\mathrm{CI}$ is slimmer, the a priori errors of the GL and the geoid heights are $10 \mathrm{~mm}$, which are both outside of the interval, meaning that either the error of the geoid is smaller than the estimated value or the GL error is smaller than its estimated value. The plot indicates that both a priori errors in Denmark are large and they both contribute to the result. The plot says, for example, if the error of the GL heights is $10 \mathrm{~mm}$, the error of the NKG2015 geoid model is between 2 $\mathrm{mm}$ and $5 \mathrm{~mm}$ within $\mathrm{CI}$ of $95 \%$.

The CIs of the error of the NKG2015 geoid model in Norway are presented in Fig. 4c. However, the interval stretches further towards a greater value of the GL. This is reasonable as the topography of Norway is rough. The plot of Norway reveals a GL height error of $15 \mathrm{~mm}$ and a geoid error of $22 \mathrm{~mm}$ it is outside the upper limit of the interval. However, it is relatively close to the upper limit, which indicates that the estimated errors are somewhat reasonable.

Fig. 4d is another similar plot but for Finland. The CIs are larger than those in the rest of countries because of the small number of the GL points. Finland is rather a flat region, which logically indicates that the error of the GL data should be relatively small so as the residuals. If we consider that the error of the GL height is $15 \mathrm{~mm}$, then the plot says that the error of NKG geoid model should be between 1 and $16 \mathrm{~mm}$, which is rather large.

One issue regarding the plots presented in Fig. 4 is the size of the CIs of the geoid error. For example, CI is larger in Finland comparing to those of Norway and Finland. This is logical as there are fewer points GL points over Finland, this causes that the degree of freedom decreases considerably. In Denmark we have about 600 GL points with very dense distribution, in addition to the flatness of the area causes that the discrepancies are all small and in the same order. The high degree of freedom on one hand and the close values of the residuals, causing a small value for the variance, on the other hand make the CI smaller. The degree of freedom is high in Norway but the values of the discrepancies are not close to each other. The CI for the geoid error is larger in Sweden than these two countries due to smaller amount of GL points.

Here, the method presented in Section 6, Eq. (34) for local estimation of the error of the geoid model based on each individual residual is presented in a CI of $95 \%$. This method is also of iterative nature and the solution has been repeated up to 50 times until it converges. The error of the geoid model reaches to about $30 \mathrm{~mm}$ in some places, mostly in Norway, and northern parts of Sweden and Finland. Small errors are observed over Denmark, south of Sweden and Finland. However, we emphasise that detrending of discrepancies plays a significant role in es- 


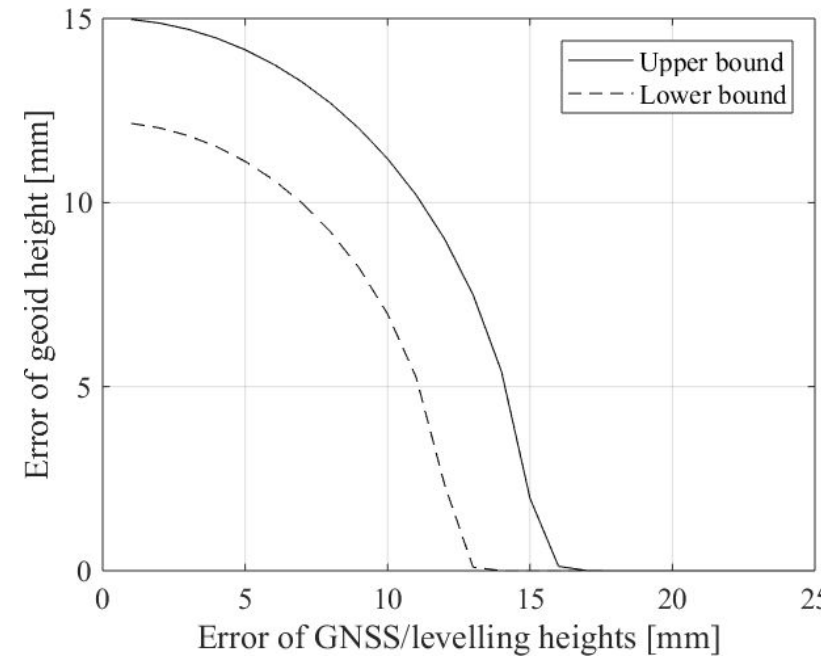

(a)

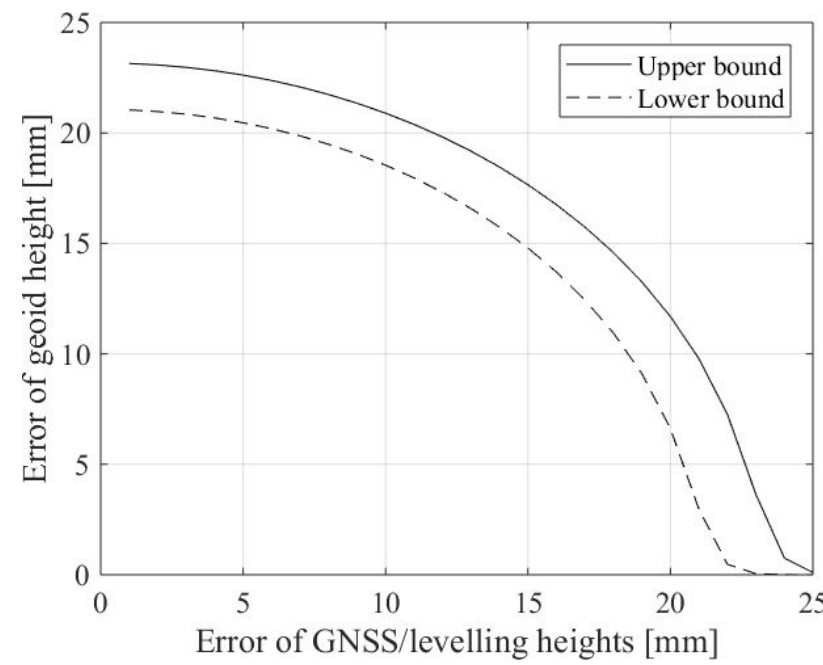

(c)

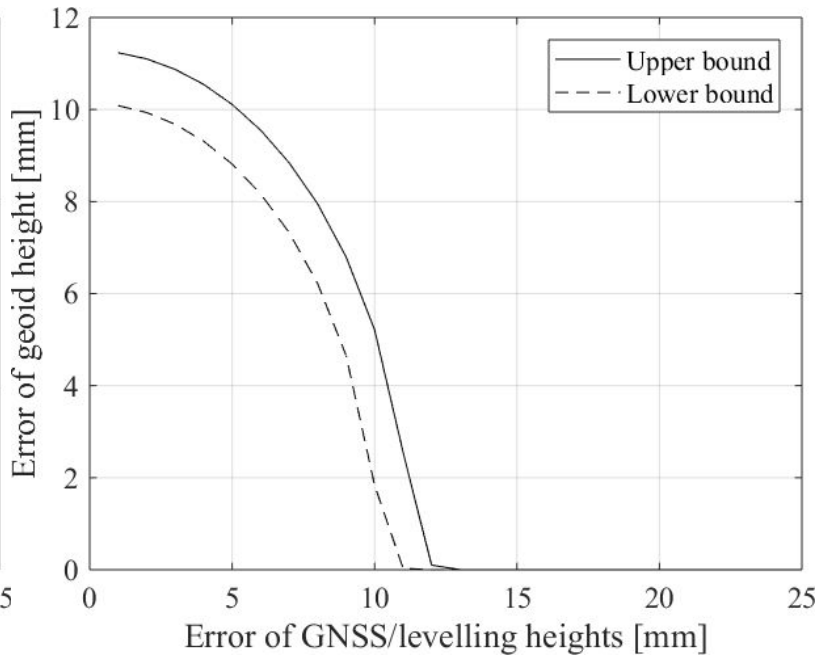

(b)

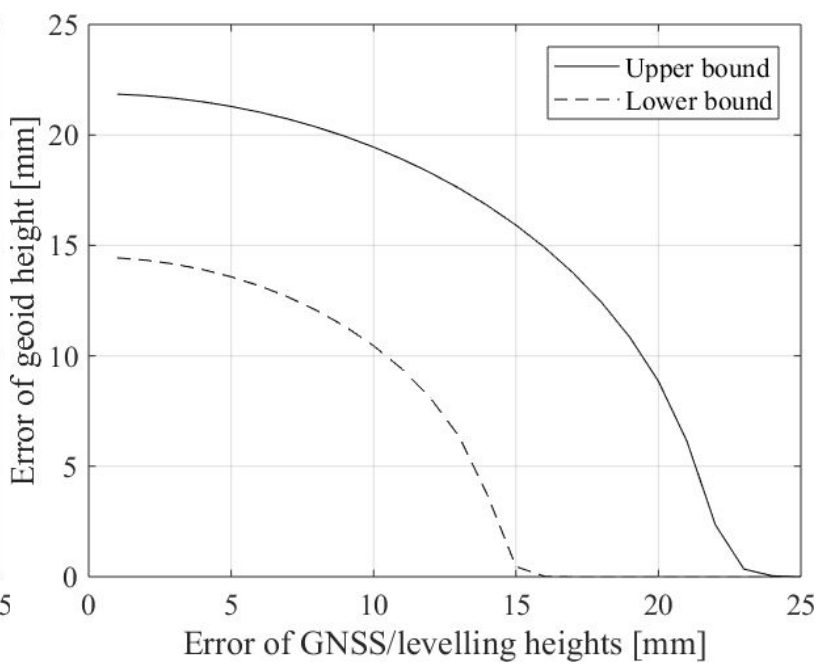

(d)

Fig. 4. The graphical relation between the Cls of the error of the NKG2015 geoid model, based on different values of error for the GL heights in a) Sweden, b) Denmark, c) Norway and d) Finland.

timation of error, and if another corrective surface model is selected other estimates will be obtained for the geoid error. Generally, a corrective surface with a limited number of parameters may be enough for detrending even if we have tested the residuals and their normalities. However, the presence of extreme values around $30 \mathrm{~mm}$ and close to zero is rather critical for the geoid model errors. The reason is that by assuming the error of GL heights to be realistic, therefore, in the estimation process the only parameter which can change is the error of geoid model to match the size of residuals and the GL height errors. Our local estimator presents small values for the geoid error in a confidence interval of $95 \%$. This is because in many places the size of discrepancies is smaller than the a priori error of the GNSS/levelling heights. However, the average value of these locally estimated errors in Sweden in about $1.8 \mathrm{~mm}$, and inside the interval presented in Table 7 showing that the error of the geoid is between 0 and $6.5 \mathrm{~mm}$. For Denmark it will be $1.3 \mathrm{~mm}$, close to the lower bound of the mentioned intervals, 1.8 and $5.2 \mathrm{~mm}$, For Norway, it is $4.4 \mathrm{~mm}$, which is quite unrealistic for such a mountainous environment. Finally, we could find an estimate for the geoid in Finland, based on the mean value of $2.8 \mathrm{~mm}$. Since the residual value at each GL point has been in applied in this approach and this value is different from one point to another there is high risk that the size of the residuals at many points are smaller than the size of the claimed error. In this case, the estimator gives a small value to the error 
of the geoid to balance it with the claimed error for the GL data and the estimated residual. This is the reason of obtaining a small average value of $4.4 \mathrm{~mm}$ for the geoid error in Norway, but we have already estimated that the error of the geoid model should be about $16 \mathrm{~mm}$ and inside the interval 14.8 and $17.7 \mathrm{~mm}$. However, we should note that the regional and the local estimators of the variance are different in their nature as well. In addition, we have considered a CI of $95 \%$ for presenting the upper bound of the local geoid errors. In the case of using a CI of $68 \%$ (one sigma), the values of the mean values derived from the local error will be larger by 2 . This means that the average geoid error in Sweden, Denmark, Norway and Finland will be, 3.6, 2.4, 8.8 and $5.8 \mathrm{~mm}$, respectively. Fig. 5 shows the map of the local geoid error based on the $\mathrm{CI}$ of $68 \%$. The map has been smoothed by a Gaussian filter for a better visualisation.

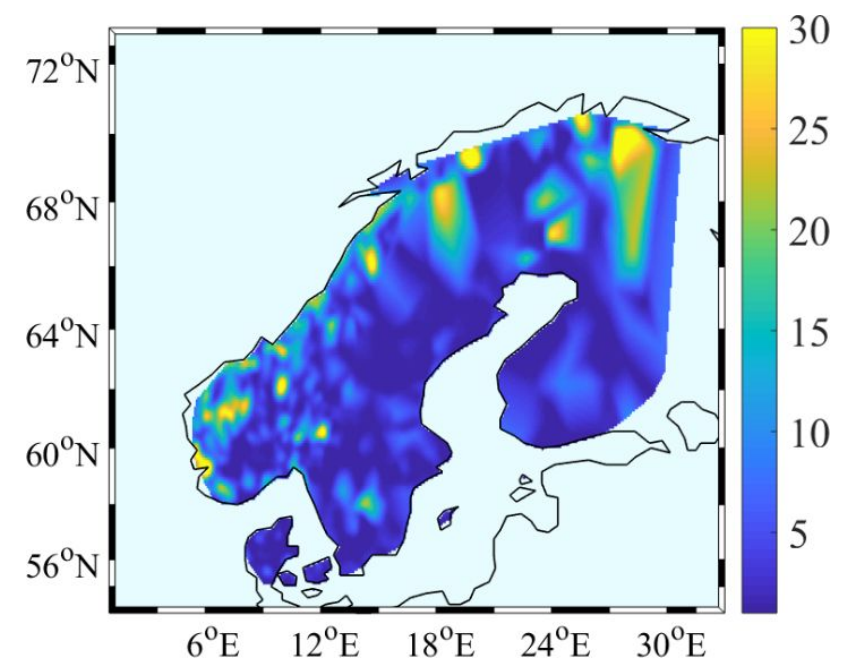

Fig. 5. The local estimates of the error of the NKG2015 geoid model with a confidence interval of $68 \%[\mathrm{~mm}]$

\section{Conclusions and recommendations}

In this study, the quality of NKG2015 geoid model has been investigated by statistical methods. A regional and a local method have been presented for estimation of variance of the geoid model and applied successfully. Two important factors for achieving proper and realistic results from these methods are to select a proper detrender or the corrective surface model for absorbing the systematic trends of the differences between the GNSS/levelling and geoid heights (discrepancies); and a priori error of the GNSS/levelling heights. If these factors are chosen improperly unrealistic estimates will be obtained for the geoid error. Here, the a priori error of the Finnish GNSS/levelling heights is $25 \mathrm{~mm}$, which significantly larger than the size of the discrepancies. In this case, the geoid error comes out 0 and unrealistic. We concluded that in case of a 4-parameter detrender, which is applied to model the systematic trends of the differences between the gravimetric and GNSS geoid heights, is suitable over Denmark and Finland, but a filtered discrepancies by a confidence interval of $95 \%$ should be used with this detrender in Sweden. A 7-parameter detrending model is suitable for the filtered discrepancies with the confidence interval of $95 \%$ in Norway. Based on the selected detrender, the confidence interval for the error of NKG2015 geoid model in Sweden, Denmark and Norway came out 0-6.5 $\mathrm{mm}, 1.8-5.2 \mathrm{~mm}, 14.8-17.7 \mathrm{~mm}$, respectively with a confidence level of $95 \%$. In addition, we estimated and plotted confidence intervals for the error of the NKG2015 geoid model based on different assumed values for the error of the GNSS/levelling height ranging from 1 to $25 \mathrm{~mm}$. The confidence interval in Finland is larger than those of other due to limited number of the GNSS/levelling points but is small for Denmark having a dense distribution of these points over a small area. Therefore, by applying the plots, the users can visually estimate the error of the NKG2015 geoid model based on the a priori value of the GNSS/heights. This is quite normal as these given errors are regional but the geoid error that we estimate is local. Our local estimator of variance for the geoid is sensitive to the size of residuals if they are smaller than the claimed error for the GNSS/levelling heights, a value of zero will be estimated for the geoid error. We found that in the case of applying this estimator to estimate a confidence interval of $68 \%$ for the local errors, the average errors of the geoid model will be inside the estimated intervals by the regional variance estimator except for Norway. Generally, this local estimator delivers average errors of 3.6, 2.4, 8.8 and $5.8 \mathrm{~mm}$ for the geoid model over Sweden, Denmark, Norway and Finland.

Acknowledgments: The authors are thankful to Docent Jonas Ågren and the Nordic Geodetic Commission, National Land Survey of Sweden and corresponding authorities in Norway, Denmark and Finland for proving the GNSS/levelling data and the NKG2015 geoid model. Professor Artu Ellmann, the handling editor of the paper and his reviewing board, is cordially acknowledged for his comments and his careful check. 


\section{References}

Abdallah A. (2009) Determination of a Gravimetric Geoid Model of Sudan Using the KTH Method, MSc thesis in Geodesy, Royal Institute of Technology (KTH), Stockholm, Sweden.

Ågren J. (2004) Regional geoid determination methods for the era of satellite gravimetry, Numerical investigations using synthetic Earth gravity models, Doctoral dissertation in Geodesy, Royal Institute of Technology, Stockholm, Sweden.

Ågren J., Sjöberg L.E., Kiamehr, R. (2009) The new gravimetric quasigeoid model KTH08 over Sweden. J. Applied Geod. 3, 143-153.

Ågren J., Strykowski G., Bilker-Koivula M., Omang O., Märdla S., Forsberg R., Ellmann A., Oja T., Liepins I., Parseliunas E., Kaminskis J., Sjöberg L.E. and Valsson G. (2016). The NKG2015 gravimetric geoid model for the Nordic-Baltic region. Presented at the 1st Joint Commission 2 and IGFS Meeting International Symposium on Gravity, Geoid and Height Systems, September 19-23 in Thessaloniki, Greece. DOI: https://doi.org/10.13140/RG.2.2.20765.20969

Bruinsma S. L., Förste C., Abrikosov O., Lemoine J.-M., , Marty J.-C., Mulet S., Rio M.-H. and Bonvalot S. (2014) ESA's satellite-only gravity field model via the direct approach based on all GOCE data, Geophys. Res. Lett., 41, 7508-7514.

Danila U. (2012) Mold2012: a new gravimetric quasigeoid model over Moldova, Licentiate thesis in Geodesy, Royal Institute of Technology (KTH), Stockholm, Sweden.

Daras I. (2008) Determination of a gravimetric geoid model of Greece using the method of KTH, M.Sc. Thesis in Geodesy and Geoinformatics Engineering, Royal Institute of Technology, Stockholm, Sweden.

Ellmann A. (2004) The geoid for the Baltic countries determined by the least squares modification of Stokes' formula, PhD thesis, Royal Institute of Technology (KTH), Stockholm, Sweden.

Ellmann A. (2005) Computation of three stochastic modification of Stokes' formula for regional geoid determination, Computers \& Geosciences 31: 742-755.

Ellmann A., Märdla S. and T. Oja (2019). The $5 \mathrm{~mm}$ geoid model for Estonia computed by the least squares modified Stokes's formula. Survey Review. DOI: https://doi.org/10.1080/00396265.2019.1583848.

ESA (1999) Gravity Field and Steady-state Ocean Circulation Mission, ESA SP-1233(1), Report for Mission Selection of the Four Candidate Earth Explorer Missions. ESA Publications, Division, p. 217 (July).

Eshagh M. (2010) Error calibration of quasi-geoid, normal and ellipsoidal heights of Sweden using variance component estimation. Contr. Geophys. Geod. 40 (1), 1-30.

Eshagh M. (2013) On the reliability and error calibration of some recent Earth's gravity models of GOCE with respect to EGM08. Acta Geod. Geophys. Hung. 48 (2), 199-208.

Eshagh M. and Sjöberg L.E. (2008) The modified best quadratic unbiased non-negative estimator (MBQUNE) of variance components. Stud. Geophys. Geod. 52, 305-320.

Eshagh M. and Zoghi S. (2016) Local error calibration of EGM08 geoid using GNSS/levelling data, Journal of Applied Geophysics 130, 209-217.

Farr T. G., Paul A. R., Edward C., Robert C., Riley D., Scott H., Michael K., Mimi P., Rodriguez E., Ladislav R., David S., Shaffer S. and
Joanne S. (2007) The Shuttle Radar Topography Mission, Reviews of Geophysics 45, RG2004, doi:10.1029/2005RG000183.

Fotopoulos G. (2003) An analysis on the calibration of geoid, orthometric and ellipsoidal height data. UCGE Report No. 20183.

Fotopoulos G. (2005) Calibration of geoid error models via a combined adjustment of ellipsoidal, orthometric and gravimetrical geoid height data. J. Geod. 79, 111-123.

Kiamehr R. (2006) Precise gravimetric geoid model for Iran based on GRACE and SRTM data and the least-squares modification of Stokes' formula with some geodynamic interpretations, Doctoral dissertation in Geodesy, Royal Institute of Technology, Stockholm, Sweden.

Kiamehr R. and Eshagh,M. (2008) Estimating variance components of ellipsoidal, orthometric and geoidal heights through the GPS/leveling network in Iran. J. Earth \& Space Phys. 34 (3), 1-13.

Märdla S., Ågren J., Strykowski G., Oja T., Ellmann A., Forsberg R., Bilker-Koivula M., Omang O., Parseliunas E., Liepins I. and Kaminskis J. (2017). From discrete gravity survey data to a high-resolution gravity field representation in the Nordic-Baltic region. Marine Geodesy 40(6): 416-453.

Pope A.J. (1976) The statistics of residuals and the detection of outliers, U.S. Dept. of Commerce, NOAA Technical Report NOS. 65 NGS 1, Rockville, Md.

Ssegendo R. (2015) A height datum for Uganda based on a gravimetric quasigeoid model and GNSS/levelling, Doctoral dissertation thesis in Geodesy, Royal Institute of Technology (KTH), Stockholm, Sweden.

Ulotu P. (2009) Geoid model of Tanzania from sparse and varying gravity data density by the KTH method, Doctoral dissertation in Geodesy, Royal Institute of Technology, Stockholm, Sweden.

Varbla S., Ellmann A., Märdla S., and Gruno A. (2017) Assessment of marine geoid models by ship-borne GNSS profiles. Geodesy and Cartography 43(2): 41-49.

Wanger, C.A., McAdoo, D.C., 2012. Error calibration of geopotential harmonics of recent and past gravitational fields. J. Geod. 86 , 99-108.

Zoghi S. (2015) On statistical test of Fennoscandian GNSS/levelling data. MSc. Thesis. Division of Geodesy and Satellite Positioning, Royal Institute of Technology (KTH), Stockholm, Sweden. 